UNIVERSIDADE DE SÃO PAULO

FACULDADE DE MEDICINA DE RIBEIRÃO PRETO

Milena Carvalho Libardi

Acurácia da Ultrassonografia Transcraniana Colorida no Diagnóstico de Forame Oval Patente 
Milena Carvalho Libardi

\section{Acurácia da Ultrassonografia Transcraniana Colorida no Diagnóstico de Forame Oval Patente}

Dissertação apresentada ao Departamento de Neurociências e Ciências do Comportamento da Faculdade de Medicina de Ribeirão Preto da Universidade de São Paulo para obtenção do título de Mestre em Ciências.

Área de concentração: Neurociências

Orientador: Prof. Dr. Octávio Marques Pontes Neto

Ribeirão Preto 
AUTORIZO A REPRODUÇÃO E DIVULGAÇÃO TOTAL OU PARCIAL DESTE TRABALHO, POR QUALQUER MEIO CONVENCIONAL OU ELETRÔNICO, PARA FINS DE ESTUDO E PESQUISA, DESDE QUE CITADA A FONTE.

O exemplar original encontra-se no Departamento de Neurociências e Ciências do Comportamento da Faculdade de Medicina de Ribeirão Preto.

\section{FICHA CATALOGRÁFICA}

\section{Libardi, Milena Carvalho}

Acurácia da Ultrassonografia Transcraniana Colorida no Diagnóstico de Forame Oval Patente, Ribeirão Preto, 2016.

52p. : il. ; $30 \mathrm{~cm}$

Dissertação de Mestrado apresentada à Faculdade de Medicina de Ribeirão Preto/USP. Área de concentração: Neurociências.

Orientador: Prof. Dr. Octávio Marques Pontes Neto

1. Acidente vascular cerebral. 2. Forame Oval Patete. 3. Ultrassonografia Transcraniana Colorida. 4. Doppler Transcraniano 


\section{FOLHA DE APROVAÇÃO}

Acurácia da Ultrassonografia Transcraniana Colorida no Diagnóstico de Forame Oval Patente.

Dissertação apresentada ao Departamento de Neurociências e Ciências do Comportamento da Faculdade de Medicina de Ribeirão Preto da Universidade de São Paulo para obtenção do título de Mestre em Ciências.

Área de concentração: Neurociências

Aprovado em:

BANCA EXAMINADORA

Prof. Dr.

Instituição: Assinatura:

Prof. Dr.

Instituição: Assinatura:

Prof. Dr.

Instituição: Assinatura: 


\section{DEDICATÓRIA}

Dedico este trabalho aos meus queridos pais Regina e Mauro pelo incentivo e convívio no amor, a todos os meus colegas de fellow e contratados da Unidade de Emergêcia do HC-FMRP-USP pelo ensino e contribuição e aos pacientes, que foram fundamentais para a realização do mesmo. 


\section{AGRADECIMENTOS}

Agradeço a todos que me acompanharam na realização deste sonho. Em especial, quero demonstrar meu profundo reconhecimento:

Agradeço ao Prof. Dr. Octavio Marques Pontes Neto, pelo voto de confiança, incentivo e competência demonstrados na orientação deste trabalho.

Aos neurologistas, Millene Rodrigues Camilo e Pedro Telles Cougo Pinto pela atenção, disponibilidade e orientações, fundamentais para este trabalho.

Aos colegas neurologistas Rui Kléber do Vale Martins Filho, Letícia Januzi Almeida Rocha, Renata da Silva Almeida Santos, Clara Monteiro Barreira, Soraia Ramos Cabette Fábio, Francisco Antunes Dias e Frederico Fernandes Alessio Alves pela disponibilidade em contribuir pelos exames de Ultrassonografia Transcraniana Colorida e Doppler Transcraniano de todos os pacientes.

Ao professor João Pereira Leite, Chefe do Departamento de Neurociências e Ciências do Comportamento da Faculdade de Medicina de Ribeirão Preto, com sua importante contribuição para o desenvolvimento desta pesquisa.

De forma muito especial, aos pacientes que participaram desta pesquisa que, mesmo diante de uma situação tão delicada, que é a internação hospitalar, se dispuseram a colaborar.

A todos, os meus sinceros agradecimentos! 


\section{EPÍGRAFE}

"Ignorance more frequently begets confidence than does knowledge: it is those who know little, not those who know much, who so positively assert that this or that problem will never be solved by science"

Charles Darwin, The Descent of Man 


\title{
RESUMO
}

\author{
Libardi, MC. Acurácia da Ultrassonografia Transcraniana Colorida no Diagnóstico de \\ Forame Oval Patente. 2016. 52f. Dissertação (Mestrado) - Faculdade de Medicina de \\ Ribeirão Preto, Universidade de São Paulo, Ribeirão Preto, 2016.
}

Introdução:O Forame Oval Patente (FOP) é a comunicação direita-esquerda (CDE) ou shunt direita-esquerda (SDE) mais comum e frequentemente encontrada em adultos jovens com Acidente Vascular Cerebral (AVC) relacionado ao mecanismo de embolia paradoxal. A Ecocardiografia Transesofágia (ETE) é considerada o padrão para visualização direta do FOP. O Doppler Transcraniano com o teste de microbolhas é frequentemente usado para detectar CDE com boa correlação com o ETE para o diagnóstico de FOP. Mais recentemente, a Ultrassonografia Transcraniana Colorida (TCCS) com inclusão do modo-B e fluxo de cor tem superado o DTC (que é realizado "as cegas") em muitas aplicações clínicas mas a acurácia do TCCS para a detecção de CDE e FOP não tem sido sistematicamente avaliada. Objetivo: Determinar se o TCCS é uma ferramenta acurada para identificar tanto FOP quanto CDE. Métodos: Foram recrutados 106 pacientes prospectivamente com Acidente Vascular Cerebral Isquêmico menores de 55 anos admitidos na Unidade de Emergência do Hospital das Clínicas da Faculdade de Medicina de Ribeirão Preto (HCFMRP). Os pacientes foram submetidos aos exames de ETE, DTC e TCCS e todos os exames incluíram a técnica do teste de microbolhas. Os examinadores foram cegos para os resultados desses exames e foi calculado a concordância Kappa de Cohen inter-examinadores para o TCCS e DTC. A acurácia para o TCCS foi calculada em comparação ao ETE. Resultados: Foram detectados CDE em 54 (50.9\%) dos pacientes (idade média $43.9 \pm 8.2$ anos) com Kappa de Cohen de 0.92 (IC 95\% 0.78-1.0) quando realizados TCCS e TCD. TEE e TCSS foram positivos em 23/98 (23.4\%) e ETE negative em 20/98 (20.4\%). Em 30 (28.3\%) o ETE revelou FOP. O TCSS teve uma sensibilidade de $88.4 \%$ (IC 95\% 0.68-0.97) e especificidade de 72.2\%(IC 95\% 0.60-0.81) e uma razão de verossimilhança positive de 3.18 (IC 95\% 2.14-4.73) para o diagnóstico de FOP. Conclusão: TCCS e DTC tiveram excelente concordância. TCCS tem uma boa acurácia para a detecção de FOP e CDE em pacientes jovens com Acidente Vascular Isquêmico.

Palavras-chave: Acidente vascular cerebral; Forame Oval Patente; Ultrassonografia Transcraniana Colorida; Doppler Transcraniano. 


\begin{abstract}
Libardi, MC. Transcranal Color Coded Sonography for detection of Patent Foramen Ovale in Young Patients with Stroke. 2016. 52f. Thesis (Master) - Faculdade de Medicina de Ribeirão Preto, Universidade de São Paulo, Ribeirão Preto, 2016.

Introduction: Patent Foramen Ovale (PFO) is the most common right-to-left shunt (RLS) and is often found in young patients with stroke related to paradoxical embolism. ContrastEnhanced Transesophageal Echocardiography (TEE) is considered a gold standard to visualize PFO. Transcranial Doppler (TCD) with bubble test is often used to detect RLS with good correlation to TEE for the diagnostic of PFO. More recently, Transcranial Color Coded Sonography (TCCS) which included B-mode and color coded imaging has overcome blind TCD in many clinical applications but the accuracy of TCCS for detection of RLS and PFO has not been systematically evaluated. Hypothesis: To determine if the TCCS is an accurate tool to identify both PFO and RLS. Methods: We investigate 106 patients with ischemic stroke under 55 years-old admitted from 2012 to 2014 in a tertiary academic hospital. Patients were evaluated with TEE, TCD and TCCS, and all exams included a saline bubble test. The examiners were blinded for the other tests results. Kappa agreement was calculated inter-examiners for TCCS and TCD. Accuracy of TCCS was calculated in comparison to TEE. Results: We detected a RLS in $54(50.9 \%)$ patients (age mean $43.9 \pm$ 8.2) with kappa agreement $0.92(95 \%$ CI $0.78-1.0)$ when performed TCCS and TCD. TEE and TCSS were positive in 23/98 (23.4\%) and TEE did not reveal contrast in 20/98 (20.4\%). In $30(28.3 \%)$ patients only TEE revealed a PFO. TCSS had a sensitivity of $88.4 \%(95 \% \mathrm{CI}$ $0.68-0.97)$, specificity of $72.2 \%$ (95\%CI $0.60-0.81)$ and positive likelihood ratio of 3.18 (2.14-4.73) of the diagnosis of PFO. Conclusion: TCCS and TCD had an excellent agreement. TCCS has a good accuracy for the detection of PFO and RLS in young patients with stroke.
\end{abstract}

Keywords: Stroke, Patent Foramen Ovale, Transcranial Color Coded Sonography, Transcranial Doppler. 


\section{LISTA DE TABELAS}

Tabela 1 -Classificação do Consenso Internacional (Quantificação da passagem de microêmbolos durante o exame de Doppler Transcraniano)

Tabela 2 - Escala Logarítmica de Spencer.

Tabela 3 - Critérios de inclusão e exclusão no estudo

Tabela 4-Classificação TOAST. Trial of Org 10172 in Acute Stroke Treatment

Tabela 5 - Perfil demográfico dos pacientes da amostra.

Tabela 6- Fatores de risco e comorbidades associadas á população do estudo.

Tabela 7 - Classificação de TOAST da população do estudo.

Tabela 8 - Número de pacientes com exame TCCS positivo/negativo e correspondência com o ETE.

Tabela 9 -Acurácia entre USTC e ETE.

Tabela 10 -Número de pacientes com exame DTC positivo/negativo e correspondência com o ETE

Tabela 11 -Acurácia entre Doppler Transcraniano (DTC) e Ecocardiografia Transesofágica (ETE)

Tabela 12-Número de pacientes com exame TCCS positivo/negativo e correspondência com o ETE.

Tabela 13 -Acurácia entre DTC e TCCS

Tabela 14 -Estudos na literatura que comprovam que a frequência de FOP na população com DCV isquêmica, no caso AVCi ou AVEi é maior no grupo controle.

Tabela 15 -Estudos que demonstram a acurácia e utilizaram diferentes soluções de contraste para o exame de DTC.

Tabela 16 -Acurácia do TCCS com o ETE em diferentes estudos. 
ACM: Artéria cerebral média

\section{LISTA DE SIGLAS}

AD: átrio direito

AE: átrio esquerdo

AIT: Ataque Isquêmico Transitório

ASA: Aneurisma de Septo Atrial

AVCi: Acidente vascular cerebral isquêmico

CDE: Comunicação Direita-Esquerda

ETE: Ecocardiografia Transesofágica

ETT: Ecocardiografia Transtorácica

DCV :doenças cerebrovasculares

DTC: Doppler Transcraniano

DM: Diabetes Mellitus

EP: embolia paradoxal

FOP: Forame Oval Patente

FSC: fluxo sanguíneo cerebral

HAS: hipertensão arterial sistêmica

HCFMRP-USP: Hospital das Clínicas da Faculdade de Medicina de Ribeirão Preto da Universidade de São Paulo

HITS: high intensity transitory signs

IC: intervalo de confiança

IQ: intervalo interquartil

MB: microbolhas

MES: sinais microembólicos

MHz: megahertz

MV: Manobra de Valsalva

NIHSS:National Institutes of Health Stroke Scale

OR:odds ratio

PA: pressão arterial

RNM: Ressonância Magnética

SAAF: Sindrome do Anticorpo Antifosfolipide

SUS: Sistema Único de Saúde

SIA: Septo interatrial

TC: Tomografia Computadorizada

TCCS: Ultrassonografia Transcraniana Colorida (Transcranial Color Coded Ultrasound)

TMB: Teste de Microbolhas

Vs: versus 


\section{SUMÁRIO}

1. INTRODUÇÃO

1.1.Forame Oval Patente: Conceito e Importância....................................................... 15

1.2 Exames Diagnósticos: FOP e CDE ………………………………............. 15

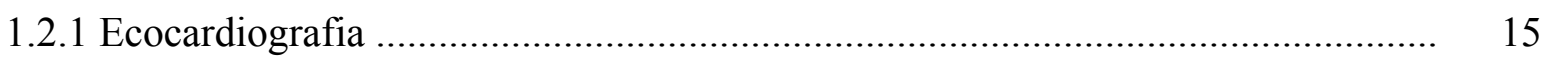

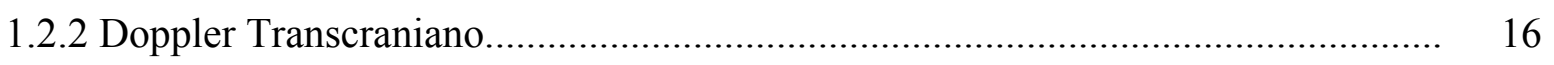

1.2.3 Ultrassonografia Transcraniana Colorida ............................................................ 19

2. OBJETIVOS........................................................................................................... 20

2.1Objetivos primários...................................................................................... 20

2.2Objetivos secundários....................................................................................... 20

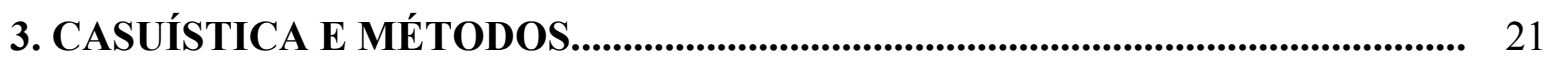

3.1 Casuística............................................................................................. 21

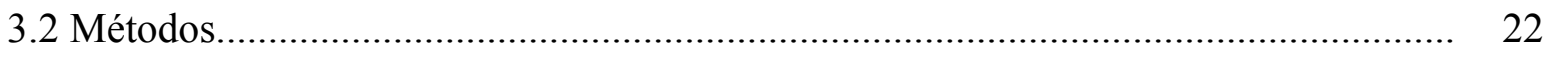

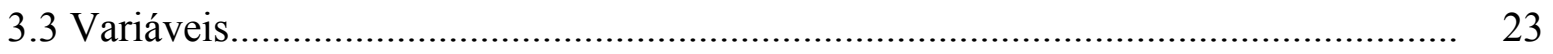

3.4 Classificação Etiologia DCV isquêmica............................................................ 23

3.5. Descrição dos modelos de Equipamentos utilizados no estudo (ETE, DTC, TCCS) 25

3.6 Pesquisa de Microêmbolos e Descrição dos exames de investigação........................... 25

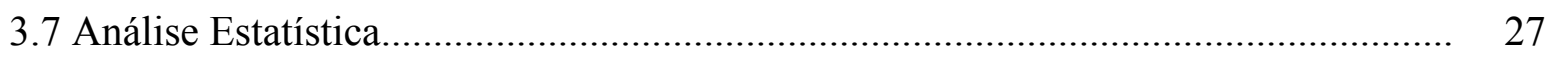

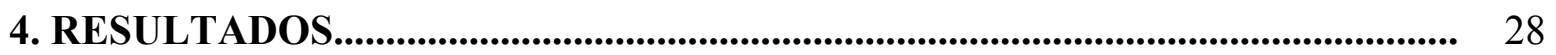

4.2 Características dos pacientes com AVC incluídos no estudo..................................... 28

4.2.1 Características demográficas e clínicas.............................................................. 28

4.2.2 Etiologia do AVC na população do estudo ............................................................ 32

4.2.3 Frequência dos exames realizados nos pacientes (TCCS, ETE, DTC)................... 33

4.2.4 TCCS vs ETE para diagnóstico de FOP ........................................................... 34

4.2.5 DTC vs ETE para o diagnóstico de FOP ........................................................... 35

4.2.6. TCCS vs DTC para o diagnóstico de CDE …………………………………...... 36 


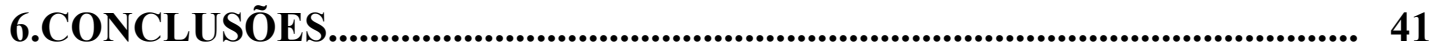

7. REFERÊNCIAS BIBLIOGRÁFICAS............................................................ 42

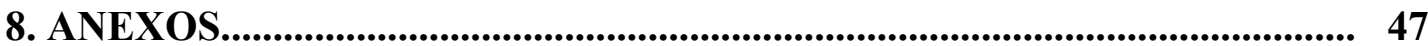

Anexo A - Termo de Consentimento ................................................................ 47

Anexo B - Trabalhos em Congresso/ Publicações em Anais ............................... 50 


\section{Introdução}

\subsection{Forame Oval Patente - Conceito e Importância}

O forame oval patente (FOP), localizado no septo interatrial (SIA) permite uma comunicação anormal entre o átrio direito $(\mathrm{AD})$ e o átrio esquerdo $(\mathrm{AE}){ }^{1}{ }^{1}$ Foi descrito pela primeira vez em 1564, pelo cirurgião italiano Leonardo Botali que o definiu como um remanescente do desenvolvimento cardíaco. Sua importância clínica está relacionada à presença de embolia paradoxal (EP), que é a passagem de substâncias originadas no sistema venoso para o sistema arterial. A EP é considerada como um possível mecanismo para o acidente vascular cerebral isquêmico (AVCi) ${ }^{2}$ e outras doenças, tais como: migrânea, doença descompressiva em mergulhadores, síndrome da ortodeóxia-platipnéia. ${ }^{3}$

O FOP é comum na população geral. Achados em estudos ecocardiográficos e autópsia indicam uma prevalência de $10 \%$ a $35 \%{ }^{4,5,6,7}$ Em populações com doença cerebrovascular isquêmica (AVCi e Ataque Isquêmico Transitório, AIT), a prevalência do FOP é maior quando comparada à população geral, particularmente em pacientes jovens com DCVi de causa desconhecida. ${ }^{8,9,10,11,12}$ Além disso, o tamanho do FOP pode ser um fator importante na fisiopatologia dos eventos cerebrovasculares, pois as maiores comunicações direita-esquerda (CDE) estão associados a um risco maior de evento isquêmico ou de sua recorrência. ${ }^{13,14,15}$.

\subsection{Exames Diagnósticos - FOP e CDE}

\subsubsection{Ecocardiografia}

Em 1969, o exame de Ecocardiograma Transtorácico (ETT) foi realizado de maneira contrastada por Gramiak e col, o que tornou possível identificar a morfologia das câmaras cardíacas e CDE in vivo. As primeiras soluções de contraste foram compostas por solução salina agitada e foi verificado a incapacidade das partículas ( maiores que 8 micrômetros) ultrapassarem a circulação pulmonar. Assim, em condições normais, não era possível atingir as câmaras esquerdas. Verificou-se também que a Manobra de Valsalva permitia a sensibilização do exame e identificação pormenorizada da CDE, já que o esforço permite aumentar o retorno venoso e a pressão no AD. Essa propriedade 
permitiu a identificação de CDE ao confirmar sua presença diretamente de maneira não invasiva. $^{7}$

Posteriormente, com avanço da tecnologia ultrassonográfica no desenvolvimento de sondas, o Ecocardiograma Transesofágico (ETE) tornou-se um método de visualização com melhor resolução da anatomia das câmaras cardíacas. O ETE é um método ultrassonográfico realizado por meio da introdução de sonda no esôfago, após anestesia local da orofaringe (para evitar o reflexo de vômito). Permite, portanto, a obtenção de informações relevantes para o esclarecimento diagnóstico de alterações estruturais e/ou funcionais do coração, com melhor sensibilidade na visualização direta do FOP quando comparado ao ETT. ${ }^{16}$

Atualmente, o ecocardiograma transesofágico (ETE) é considerado método padrãoouro para o diagnóstico do FOP e da EP, pois permite a visualização direta da CDE. O ETE permite a identificação de microbolhas (MB), compostas por soluções que não cruzam o sistema capilar pulmonar em condições normais, injetadas em veia periférica. ${ }^{16}$ Apesar desta característica, o ETE é um método operador-dependente e com limitações por não permitir com segurança a identificação da comunicação extracardíaca. Outra desvantagem, é a invasibilidade do método pelo uso de uma sonda esofágica causando desconforto e necessidade de sedação. Em pacientes com AVCi, que muitas vezes possuem disfagia e afasia, a limitação á deglutição é outra desvantagem ao exame, não permite o posicionamento ideal do transdutor, bem como a capacidade do paciente em cooperar com a MV, que sensibiliza a passagem de microbolhas em FOP de tamanhos menores. ${ }^{17,18}$

\subsubsection{Doppler Transcraniano (DTC)}

O DTC é exame ultra-sonográfico sem imagem, em modo Doppler pulsado, operando a baixas freqüências, que obtém dados em tempo real, permitindo a avaliação da direção e velocidade do fluxo sanguíneo nas artérias cerebrais basais, por meio de desvios na freqüência da onda sonora refletida pelo sangue em movimento. O transdutor deve ser posicionado nas denominadas janelas ósseas, que podem ser forames naturais ou áreas do crânio onde a díploe tem espessura reduzida, como ocorre na região temporal. Este método foi primeiramente descrito por Aaslid et al. ${ }^{1}$, em 1982, tendo ocorrido crescente desenvolvimento tecnológico e científico em suas aplicações nas últimas duas décadas.As principais vantagens do DTC são portabilidade, inocuidade e relativo baixo custo. Como todo 
método ultra-sonográfico, é dependente do conhecimento e habilidade do operador tanto na realização quanto na interpretação dos resultados.

O DTC tem sido utilizado como exame de triagem na investigação de FOP previamente ao ETE por ser não-invasivo, de fácil realização e repetição e ainda apresenta alta precisão quando comparado ao ETE no diagnóstico de embolia paradoxal e FOP. ${ }^{19,20}$ Para a pesquisa de FOP pelo DTC são utilizadas soluções contrastantes e técnicas sensibilizadoras semelhantes às utilizadas com o ETE, sendo que a identificação do FOP ocorre pela visualização de sinais transitórios de alta intensidade (high intensity transitory signs - HITS) ou sinais microembólicos (MES) durante a monitoração de determinado vaso cerebral (em geral, a artéria cerebral média - ACM). Tais sinais, quando presentes, devem estar dentro do ciclo cardíaco e espectro de onda, com representação sonora característica (pop, estalido), possuem curta duração (geralmente 0,1 segundo), alta intensidade ( 3 decibéis acima do basal) e unidirecionais. ${ }^{3}$

A configuração do tronco supra-aórtico e o maior fluxo sanguíneo fazem com que a maioria das embolias encefálicas ocorra em direção à circulação carotídea, portanto o principal vaso insonado no estudo de EP é a artéria cerebral média (ACM), considerada a terminação da artéria carótida interna e principal vaso da circulação anterior ${ }^{21}$, embora outras artérias intracranianas possam ser insonadas para este propósito. A ACM é insonada através da janela transtemporal e pode ser monitorada bilateralmente através do uso de capacete apropriado. ${ }^{22,23}$

Assim, para a pesquisa de MES através do DTC, critérios diagnósticos foram estabelecidos em Consensos Internacionais. Existem duas classificações utilizadas para categorizar os MES detectados. Inicialmente, em 1998, foi estabelecido o Consenso Internacional $^{29}$ que classificou o número de sinais sendo positivo se pelo menos um sinal for observado. Esta classificação não possui fins prognósticos ou correlação com o tamanho da comunicação/forame oval (Tabela 1). 
Tabela 1. Classificação do Consenso Internacional - Quantificação da passagem de microêmbolos durante o exame de Doppler Transcraniano. ${ }^{29}$

\begin{tabular}{|c|}
\hline Classificação do Consenso Internacional \\
\hline 0 microêmbolos (resultado negativo) \\
\hline $1-10$ microêmbolos \\
\hline$>10$ microêmbolos \\
\hline$>25$ microêmbolos (padrão chuveiro) \\
\hline Incontáveis microêmbolos (padrão cortina) \\
\hline
\end{tabular}

Já em 2004, foi elaborada uma escala chamada de Escala Logaritmica de Spencer que avaliou a quantidade de MES bilateralmente através de registro em Modo-M Power. Esta escala apresentou, quando comparada aos critérios do Consenso Internacional, uma maior especificidade. Ao considerar um cut-off de 30 sinais, grau III de Spencer, houve redução no número de falsos positivos e também houve relação com comunicação de maior tamanho $(>4 \mathrm{~mm})$, que inclusive está associada ao aumento do risco ou recorrência de AVCi (Apêndice 2). ${ }^{24-29}$

Tabela 2. Escala Logarítmica de Spencer. ${ }^{27}$

\begin{tabular}{|lc|}
\hline Escala Logarítmica de Spencer \\
\hline Grau 0: & 0 microêmbolos (resultado negativo) \\
\hline Grau I: & $1-10$ microêmbolos \\
\hline Grau II: & $11-30$ microêmbolos \\
\hline Grau III: & $31-100$ microêmbolos \\
\hline Grau IV: & $100-300$ microêmbolos \\
\hline
\end{tabular}

No entanto, uma limitação importante no uso do DTC isolado é a ausência de janela óssea temporal observada em aproximadamente $6 \%$ a $18 \%$ da população. ${ }^{3,18,30-32}$ Nestes casos, a insonação da artéria basilar ou da artéria carótida interna extracraniana pode ser utilizada para identificar a presença de HITS ou MES, apresentando sensibilidade e especificidade de até $100 \%$ quando comparadas a insonação da ACM, devido a presença de fenômeno embólico nestes territórios. ${ }^{22,28 .}$

Outra limitação do DTC é a de que o exame é realizado "ás cegas”, ou seja, a artéria cerebral média é insonada de acordo com a profundidade, espectro de onda e som característico e exige, portanto aparelho específico e examinador bem treinado. ${ }^{32,33}$ 


\subsubsection{Ultrassonografia Transcraniana Colorida (TCCS)}

O uso da Ultrassonografia Transcraniana Colorida (TCCS) ou Duplex Scan Colorido, foi implantada recentemente como ferramenta adicional para o diagnóstico de doenças cerebrovasculares (DCV). ${ }^{18,32,34}$ A técnica permite a obtenção de imagem bidimensional da vasculatura cerebral combinada ao modo Doppler, isto é do modo-B, com uma visualização direta da circulação intracraniana. Alguns estudos demonstraram que tal ferramenta pode permitir identificar corretamente a artéria cerebral média e distingui-la de outros grandes vasos intracranianos, particularmente a artéria cerebral posterior ipsilateral, aumentando a contagem HITs ou MES nesses territórios. Além disso, em alguns casos, nos quais há uma janela óssea inadequada ao DTC isolado, o exame pode ser feito com o TCCS, que eventualmente é capaz de possibilitar algum sinal para a insonação do vaso. ${ }^{18,32}$

A vantagem desse método, quando comparado ao DTC, é que pode ser feito em aparelhos convencionais de ultrassonografia, desde que um transdutor de $2 \mathrm{Mhz}$ seja acoplado e o software permita acesso a circulação intracraniana. Por conseguinte, a disponilidade do método é maior e desproporcional ao seu custo. A técnica utilizada para a pesquisa de MES no TCCS é a mesma para o DTC.

Por conseguinte, ao considerar que uma grande proporção da população possui FOP e que muitos pacientes com AVCi de causa indeterminada podem portar a CDE, o seu correto diagnóstico poderá proporcionar intervenções capazes de prevenir novos eventos isquêmicos e um manejo terapêutico adequado.

Embora existam relatos do ETE e do DTC na literatura para o diagnóstico de FOP, tais exames ainda possuem fatores limitantes, o que pode deixar de diagnosticar FOP em alguns casos. A acurácia do TCCS para vasos intracranianos e cervicais ainda é pouco estudada sistematicamente na literatura na investigação de FOP. Sendo assim, tal exame possibilitará a triagem de um maior número de casos de forame oval patente e, portanto, permitir maiores orientações específicas para tentar evitar recidiva da DCV. Neste contexto, é importante caracterizar melhor a utilidade do TCCS para investigação etiológica de adultos jovens que sofreram um AVCi ou AIT, visto que esse grupo possue maior prevalência de FOP, comparando com a população em geral, além de menor incidência de fatores de risco convencionais (hipertensão arterial sistêmica (HAS), Diabetes Mellitus (DM), problemas cardíacos e dislipidemia. 


\section{Objetivos}

\section{OBJETIVOS PRIMÁRIOS}

1. Verificar a acurácia da Ultrassonografia Transcraniana Colorida (TCCS) para o diagnóstico de FOP, em pacientes jovens com acidente vascular cerebral isquêmico (AVCi) ou ataque isquêmico transitório (AIT), quando comparado a Ecocardiografia Transesofágica ETE (padrão- ouro).

2. Verificar a concordância da Ultrassonografia Transcraniana Colorida (TCCS) para o diagnóstico de $\mathrm{CDE}$, em pacientes jovens com acidente vascular cerebral isquêmico (AVCi) ou ataque isquêmico transitório (AIT), quando comparado ao Doppler Transcraniano (DTC).

\section{OBJETIVOS SECUNDÁRIOS}

1. Descrever as características demográficas e os fatores de risco da população jovem com AVCi e AIT admitidos em uma unidade de emergência terciária de referência para AVC, diagnosticados com ou sem FOP em sua investigação etiológica. 


\section{Casuística e Métodos}

\section{1 - Casuística}

Este estudo foi realizado prospectivamente com uma amostra populacional de pacientes registrados e admitidos na Unidade de Emergência do HCFMRP-USP. Todos os pacientes, no momento da inclusão no estudo, tinham o diagnóstico de Doenças Cerebrovasculares (DCV) isquêmica (AVCi ou AIT).

$\mathrm{O}$ estudo foi analisado e aprovado pelo Comitê de Ética em Pesquisa (CEP) do HCFMRP-USP, de acordo com o número do Certificado de Apresentação para Apreciação Ética (CAAE) 33049314.7.0000.5440 e processo HCRP n055576/2014.

O termo de consentimento livre e esclarecido (Anexo A) foi assinado pelo paciente ou seu responsável legal antes da inclusão no estudo.

Foram avaliados, prospectivamente, os pacientes jovens admitidos na Unidade de Emergência do HCFMRP-USP, de acordo com os critérios de inclusão e exclusão (Tabela 3) no período de Janeiro a Dezembro de 2014. A pesquisa também coletou dados epidemiológicos e exames de adultos jovens com DCV isquêmica internados de Janeiro de 2012 a Dezembro de 2013 e esses pacientes foram recrutados de maneira prospectiva e consentida para a realização do TCCS e/ou DTC. Tais exames já seriam realizados para complemento da investigação da etiologia do AVCi ou AIT.

Tabela 3. Critérios de inclusão e exclusão no estudo

\section{CRITÉRIOS DE INCLUSÃO}

- $\quad$ Idade $>$ ou igual a 18 anos e < ou igual a 55 anos com indicação de investigação de FOP

- Acidente vascular cerebral isquêmico ou ataque isquêmico transitório

\section{CRITÉRIOS DE EXCLUSÃO}


- Pacientes que já tenham realizado o procedimento para fechamento do FOP

- Impossibilidade de realização de um dos exames

- Ausência de janela transtemporal adequada para insonação da artéria cerebral média.

- Não assinatura do termo de consentimento informado de acordo com comitê de ética do HCFMRP-USP

\section{2 - Métodos}

Trata-se de um estudo observacional, analítico e cross-seccional, realizado de maneira prospectiva. No período da pesquisa, foram incluídos 56 pacientes com idade entre 18 a 55 anos de Janeiro a Dezembro de 2014 com indicação de investigação de FOP, internados na Unidade de Emergência do HCFMRP-USP com diagnóstico de DCV isquêmica. No período da internação hospitalar, esses pacientes foram submetidos a investigação básica da etiologia do AVCi ou AIT conforme protocolo do serviço (Tomografia Computadorizada de Crânio admissão e controle e/ou Ressonância Magnética Cerebral, estudo de vasos intracranianos e cervicais pela Angiotomografia e/ou Angiorresonância, DTC e/ou TCCS, ETT e/ou ETE, exames laboratoriais como: lipidograma, função renal, glicemia, hemoglobina glicosilada, enzimas hepáticas, enzimas cardíacas, eletrólitos, sorologias para doença de Chagas e Sifílis (VDRL). Exames de investigação em paciente jovem com DCV isquêmica também foram colhidos nesses pacientes (Provas de Atividade inflamatórias, Rastreio para Vasculite e Doenças Imunomediadas, Trombofilias Arteriais e Venosas, Sorologias para HIV, Hepatites do tipo B e C). Tais exames foram também complementados ambulatorialmente após alta hospitalar.

No mesmo período do estudo, foram recrutados 61 pacientes com idade entre 18 a 55 anos que estiveram em internação hospitalar entre Janeiro de 2012 e Dezembro de 2013 com diagnóstico de AVCi ou AIT. Foram coletados resultados dos exames laboratoriais séricos e de liquor, dados demográficos e presença de fatores de risco (HAS, DM2, dislipidemia, tabagismo, etilismo, uso de anticoncepcional em mulheres, presença de migrânea com e sem aura), história de trombofilias, tromboses venosas e arteriais prévias, vasculites, sorologias para Chagas, Sifilis, Hepatites, HIV além do resultados de exames de DTC, TCCS e ETE para pesquisa de FOP ou CDE. 
Os exames de DTC e TCCS de todos os pacientes incluídos foram realizados pela equipe de Neurologia Vascular do HCFMRP-USP e ambos os exames foram realizados na Unidade de Emergência da instituição. O exame de TCCS foi executado de maneira independente do DTC, por investigadores diferentes e cegos para um dos exames.

\subsection{Variáveis}

Foram coletadas as seguintes variáveis: idade, sexo, etnia, tipo de DCV isquêmica (AVCi ou AIT), presença das seguintes comorbidades: HAS, DM2, dislipidemia, migrânea (com e sem aura), doença de Chagas, história prévia de evento cerebrovascular, histórico de trombose venosa ou arterial em qualquer sítio, antecedente pessoal de neoplasias. Foram realizados exames de Trombofilias Arteriais e Venosas ( SAAF com anticardiolipinas, dosagem de complemento C3 e C4, Deficiência de Proteínas C e S, dosagem de antitrombina III, mutação do fator V de Leiden e gene da protrombina 20210, dosagem de homocisteína), rastreio para Vasculites e doenças imunomediadas e provas de atividade inflamatórias (Líquor com hiperproteinorraquia, proteína C reativa, velocidade de hemossedimentação , alfa-1 glicoproteína, fator anti-núcleo, dosagem de anticoagulante lúpico), sorologias (Chagas, Sifilis, HIV, Hepatite B e C), dosagem de ácido fólico e vitamina B12, historia de consumo de drogas ilícitas, toxicológico (quando pertinente), consumo de cigarro (histórico de tabagismo) e consumo de bebida alcoólica (histórico de etilismo). Para mulheres, foram analisados históricos de uso de anticoncepcionais orais e injetáveis, histórico de abortos, histórico gestacional prévio e puerperal, terapia de reposição hormonal. Para análise dos resultados, os exames referentes a Trombofilias Arteriais e Venosas foram analisados em um grupo considerado Trombofilias, tal como presença ou ausência de Vasculite.

\subsection{Classificação Etiologia DCV isquêmica}

Para a classificação da etiologia da DCV isquêmica dos pacientes do estudo, foi utilizado o critério TOAST ${ }^{35}$ (tabela 4). Na categoria indeterminado, foram alocados aqueles pacientes cuja investigação estava incompleta e não poderia ser determinada e aqueles pacientes cuja investigação foi completada, porém sem etiologia definida (chamados de criptogênicos). A presença ou ausência do FOP ou de qualquer CDE é independente dessa classificação.

O critério TOAST é definido em cinco categorias: ${ }^{35}$ 
3.4.1. Aterosclerose de grandes artérias ou grandes vasos: Engloba casos em que existe o acometimento de grandes vasos ou de seus ramos intracranianos presumivelmente devido a aterosclerose, assim exames de imagem (Arteriografia, Angiotomografia, Angioressonância e Doppler cervical e transcraniano) são sugestivos de estenose $>50 \%$ no território envolvido. Evidência clínica de envolvimento cortical (afasia, negligência), tronco cerebral ou cerebelo e história de claudicação intermitente, AIT em mesmo território vascular, sopro cervical são características dessa categoria. As lesões isquêmicas devem ser maiores que $15 \mathrm{~mm}$ e podem ser corticais ou cerebelares.

3.4.2. Doença de pequenas artérias ou pequenos vasos (lacuna): Essa categoria é definida pela presença clínica de síndrome lacunar, história de HAS e DM, ausência de envolvimento cortical e presença de infarto menor que $15 \mathrm{~mm}$ (mais comumento no território dominante das pequenas artérias perfurantes) ou imagem de tomografia ou ressonância normais.

3.4.3. Cardioembolismo: Essa categoria inclui pacientes com oclusões arteriais por êmbolos de origem cardíaca. O critério TOAST define fontes cardioembólicas de alto risco e médio risco baseadas na propensão relativa de embolia. Assim, fontes de alto risco englobam pacientes com prótese valvar metálica, presença de fibrilação atrial com ou sem estenose mitral, presença de trombo intracavitário, síndrome do nó sinusal, infarto do miocárdio com menos de 4 semanas, miocardiopatia dilatada, parede acinética de ventrículo esquerdo, mixoma atrial e endocardite bacteriana. Fontes de médio risco incluem prolapso de válvula mitral, calcificação ou estenose mitral, turbulência atrial, aneurisma de septo atrial, hipocinesia de parede de ventrículo esquerdo, FOP e infarto do miocárdio entre 4 semanas e 6 meses. Evidência clínica de isquemia em mais de um território arterial, acometimento cortical e presença de fontes de alto risco são características dessa categoria. Fontes de médio risco e sem outra causa são consideradas como possível cardioembolismo, mas devem ser classificadas na categoria indeterminada até investigação completa que sugira um alto risco para cardioembolia.

3.4.4. Outras causas: Essa categoria inclui pacientes com vasculopatias não ateroscleróticas, estados de hipercoagulabilidade e distúrbios hematológicos. Toda investigação para fonte embólica e causa aterosclerótica deve ser excluída. 
3.4.5. Indeterminado: Quando a causa do AVCi ou AIT não pode ser determinada com algum grau de confiança. Alguns pacientes não tem etiologia definida a despeito de exaustiva investigação. Essa categoria também engloba pacientes com dois ou mais mecanismos de AVCi ou AIT (por exemplo: paciente com síndrome lacunar e estenose carotídea).

Tabela 4. Classificação TOAST. Trial of Org 10172 in Acute Stroke Treatment (TOAST trial), Stroke, 1993.

Aterosclerose de grandes artérias ou grandes vasos

Doença de pequenas artérias ou pequenos vasos (lacuna)

Cardioembolismo

Outras Causas

Indeterminado

3.5 Descrição dos modelos de Equipamentos utilizados no estudo (ETE, DTC, TCCS)

Para a investigação diagnóstica de FOP e CDE nos pacientes do estudo, foram utilizados os seguintes equipamentos (marcas e modelos elencados abaixo):

$\checkmark$ Ecocardiograma Transesofágico (ETE) modelo VIVID GE (Cardiologia)

$\checkmark$ Doppler Transcraniano (DTC) marca Nicolet, modelo EME Trans-Scan 3D (Überlingen, Germany), equipado com um transdutor de $2 \mathrm{MHz}$. A artéria cerebral média é insonada numa profundidade em torno de 50-60 milímetros.

$\checkmark$ Ultrassonografia Transcraniana Colorida (TCCS) Duplex Toshiba Xario modelo SSA-660A, equipado com transdutor de $2 \mathrm{MHz}$. A artéria cerebral média é insonada no ponto em que ela é visualizada no monitor após o sifão carotídeo e a porção distal da artéria carótida interna.

\subsection{Pesquisa de Microêmbolos e Descrição dos exames de investigação}

\subsubsection{TCCS e DTC}

A pesquisa de MES ou verificação de HITS adota a mesma técnica para ambos os exames de TCD e TCCS. A técnica padronizada em nosso laboratório é realizada dos critérios internacionais ${ }^{19}$. Esta técnica de exame consiste em posicionar o paciente em decúbito supino, em uma sala com temperatura controlada entre $24^{\circ}$ e $28^{\circ} \mathrm{C}$ e insonar a artéria cerebral média do lado contralateral ao acidente vascular cerebral, através da janela temporal com profundidade entre 50-60 mm. Com a artéria insonada, é injetada, em veia 
antecubital de membro superior direito, uma solução de $9 \mathrm{ml}$ de soro físiológico $0,9 \%$ e $1 \mathrm{ml}$ de ar, previamente agitada entre duas seringas por meio de torneira de três vias. Este teste, conhecido como teste de microbolhas (TMB) e é realizado com o paciente em repouso e sensibilizado pela Manobra de Valsalva (MV), a qual é iniciada após 5 segundos da injeção da solução. Caso não sejam detectados sinais microembólicos, o teste em repouso é mais uma vez repetido e então associado à manobra de Valsalva. O número de MES ou HITs é registrado em contagem manual conforme o aparecimento dentro do ciclo cardíaco e as seguintes características:

- Curta duração (abaixo de 0,1 seg)

- Presença de som audível típico (“chirps”)

- Alta intensidade dentro do espectro de fluxo de Doppler;

- Visualização em qualquer momento do ciclo cardíaco;

- Unidirecional.

O tempo de passagem da solução da veia cubital até a artéria cerebral média é em torno de 11 segundos quando há comunicação intracardíaca e em torno de 14 segundos, quando pulmonar. ${ }^{19}$ No entanto, este tempo deve somente orientar a monitorização, e não inferir a topografia da comunicação circulatória, se presente. ${ }^{29}$

Portanto, a positividade para MES caracterizará a presença da CDE, que será quantificado de acordo com o Consenso Internacional e Classificação de Spencer (tabela 1 e tabela 2). ${ }^{19,27}$

Os pacientes com pesquisa positiva no teste de sinais microembólicos ou presença de HITS serão considerados portadores de CDE. Pelo Consenso Internacional, será considerado FOP pequeno, a passagem de até 10 HITS ou microêmbolos e FOP grande, a passagem de > 10 HITS ou microêmbolos.

Os exames foram realizados com pelo menos 2 monitorizações em repouso e $2 \mathrm{em}$ MV na ACM. Como padronização do laboratório de nosso serviço, a ACM direita é insonada na janela transtemporal em ambos os exames. Não foram registradas intercorrências em nenhum dos exames em relação a efeitos adversos da punção venosa periférica ou da injeção da solução salina agitada. Todos os exames foram realizados com a técnica descrita acima por ao menos dois neurossonologistas, cegos para um dos exames a fim de estabelecer a concordância do método.

\subsubsection{Ecocardiograma Transesofágico (ETE)}


Como citado previamente, o ETE é realizado de forma rotineira em nosso serviço para pacientes com indicação de pesquisa de FOP (AVCi e AIT em adulto jovem).

O ETE foi realizado por dois médicos cardiologistas sem conhecimento prévio do resultado do DTC e/ou TCCS. A técnica para o exame consiste em aquisição de imagens registradas por meio de transdutor de $8 \mathrm{MHz}$ presente na extremidade da sonda esofágica. Para introdução da sonda esofágica, foi utilizado anestesia local da orofaringe com lidocaína spray $10 \%$ e gel $2 \%$. A sedação foi opcional, para conforto do paciente, sendo habitualmente de grau leve a moderado e realizada com benzodiazepínico, preferencialmente midazolam intravenoso. O exame foi realizado em jejum de pelo menos 4 horas e o setor de cardiologia realiza o exame com a exigência do termo de consentimento do paciente ou responsável.

Nos pacientes que participaram da pesquisa e foram submetidos ao ETE, o exame foi laudado por 2 cardiologistas sem conhecimento prévio do resultado do DTC e/ou do TCCS.

\subsection{Análise estatística}

Para a análise estatística descritiva foram utilizadas medidas de tendência central e dispersão. A estatística inferencial foi realizada através do Teste Exato de Fisher, teste QuiQuadrado para variáveis categóricas e teste t-student para variáveis contínuas e comparativa das proporções entre as variáveis qualitativas. O nível de significância estatística para todas as análises foi de $5 \%$ bicaudal.

A acurácia dos testes diagnósticos foi verificada considerando a sensibilidade, especificidade, valor preditivo positivo e valor preditivo negativo, likelihood ratio ou razão de verossimilhança (positivo, negativo) e cálculos das probabilidades pré e pós teste. A concordância entre os métodos diagnósticos para pesquisa de CDE (DTC e TCCS) foi medida utilizando o coeficiente de concordância Kappa de Cohen, considerando uma concordância quase perfeita ou excelente valores maiores que 0.81 .

O Programa estatístico utilizado para esse estudo foi o SPSS versão 20 (IBM INC., ARMONK, NY,EUA). 


\section{Resultados}

\section{2 - Características dos pacientes com AVC incluídos no estudo}

\subsection{1 - Características demográficas e clínicas}

Durante os 12 meses de realização do estudo, foram incluídos 56 pacientes de maneira prospectiva internados em 2014 e 61 pacientes que estiveram em consulta ambulatorial para seguimento e foram internados no período entre 2012 e 2013, convocados de maneira consentida e prospectiva com indicação de pesquisa de FOP para realizarem os exames de TCCS. Ao todo 117 pacientes compuseram a casuística inicial do estudo. Todos tinham exames de imagem (Tomografia Computadorizada do Crânico e/ou Ressonância Magnêtica do Encéfalo) que confirmaram DCV isquêmica e/ou história de déficit neurológico agudo condizente com AVCi e/ou AIT. No entanto 11 pacientes foram excluídos do estudo: 4 pacientes que desistiram após a inclusão, 5 pacientes sem janela transtemporal adequada para a realização de DTC e/ou TCCS, 2 pacientes que não possuíam exames de ETE, DTC e/ou TCCS até a data de fechamento da coleta. Assim, tivemos 54 pacientes incluídos em 2014 e 52 pacientes que tiveram passagem no serviço de emergência do HCFMRP-USP, totalizando 106 pacientes na amostra final desta análise.

Este grupo foi constituído por 43 (40,5\%) homens e 63 (59.4\%) mulheres. A idade média foi de 44,6 \pm 8.5 anos, com predomínio de brancos (71,7\%). A prevalência de CDE na amostra do estudo foi de 54 (50.9\%). Dos pacientes, 99 (93.4\%) tiveram o diagnóstico de AVCi e somente 7 (6.6\%) tiveram AIT. A tabela 5 demonstra as características da população do estudo de acordo com a presença e ausência de CDE. 
Tabela 5. Perfil demográfico dos pacientes da amostra.

\begin{tabular}{|c|c|c|c|c|}
\hline \multirow[b]{2}{*}{ Variável } & \multirow[b]{2}{*}{$\begin{array}{c}\text { total } \\
\mathrm{n}=106\end{array}$} & \multicolumn{2}{|c|}{$\begin{array}{c}\text { Comunicação Direita-Esquerda } \\
\text { (CDE) }\end{array}$} & \multirow[b]{2}{*}{$\mathrm{p}$ value } \\
\hline & & $\begin{array}{l}\text { Sim } \\
n=54\end{array}$ & $\begin{array}{c}\text { Não } \\
\mathrm{n}=52\end{array}$ & \\
\hline Idade, média \pm DP & $44.6 \pm 8.5$ & $43.9 \pm 8.2$ & $45.3 \pm 8.7$ & \\
\hline \multicolumn{5}{|l|}{ Sexo } \\
\hline Masculino, n (\%) & $43(40.5)$ & $18(33.3)$ & $25(48)$ & 0.16 \\
\hline Feminino ,n (\%) & $63(59.4)$ & $36(66.7)$ & $27(51.9)$ & \\
\hline Etnia & & & & 0.70 \\
\hline Branco, n (\%) & $76(71.7)$ & $39(72.2)$ & $37(71.1)$ & \\
\hline Negro ,n (\%) & $24(22.6)$ & $11(20.3)$ & $13(25)$ & \\
\hline Outros, n (\%) & $6(5.6)$ & $4(7.4)$ & $2(3.8)$ & \\
\hline \multicolumn{5}{|l|}{ Doença } \\
\hline \multicolumn{5}{|l|}{ Cerebrovascular } \\
\hline $\mathrm{AVCi}, \mathrm{n}(\%)$ & $99(93.4)$ & $52(96.2)$ & $47(90.3)$ & 0.26 \\
\hline AIT , n (\%) & $7(6.6)$ & $2(3.7)$ & $5(9.6)$ & \\
\hline
\end{tabular}

Os fatores de risco mais relatados foram: hipertensão arterial sistêmica (56.6\%), tabagismo (45.3\%) e dislipidemia (31.1\%). As principais características clínicas e variáveis dos pacientes segundo critérios de inclusão/exclusão do estudo, estão descritas na tabela 6 . 
Tabela 6. Fatores de risco e comorbidades associadas á população do estudo

\begin{tabular}{|c|c|c|c|c|}
\hline \multirow[b]{4}{*}{ Fatores de Risco } & \multicolumn{4}{|c|}{ Comunicação Direita } \\
\hline & \multirow[b]{3}{*}{$\begin{array}{c}\text { Total } \\
\mathrm{n}=106\end{array}$} & \multirow{2}{*}{\multicolumn{2}{|c|}{$\begin{array}{c}\text { Esquerda } \\
\text { CDE }\end{array}$}} & \multirow[b]{3}{*}{$\mathrm{p}$ value } \\
\hline & & & & \\
\hline & & $\begin{array}{c}\text { Sim } \\
\mathrm{n}=54\end{array}$ & $\begin{array}{c}\text { Não } \\
\mathrm{n}=52\end{array}$ & \\
\hline Etilismo & $20(18.8)$ & $11(20)$ & $9(17)$ & 0.80 \\
\hline Tabagismo & $48(45.3)$ & $23(42)$ & $25(48)$ & 0.69 \\
\hline Obesidade & $20(18.8)$ & $9(16)$ & $11(21)$ & 0.62 \\
\hline Hipertensão Arterial & $60(56.6)$ & $25(46.2)$ & $35(67)$ & 0.03 \\
\hline Diabetes Mellitus & $20(18.8)$ & $7(12.9)$ & $13(25)$ & 0.13 \\
\hline Dislipidemia & $33(31.1)$ & $15(27)$ & $18(34.6)$ & 0.53 \\
\hline Migrânea & $20(18.8)$ & $13(24)$ & $7(13.4)$ & 0.21 \\
\hline Doença de Chagas & $3(2.8)$ & $2(3.7)$ & $1(1.9)$ & 1.0 \\
\hline Anticoncepcional & & & & \\
\hline ou Terapia Hormonal & $11(10.3)$ & $9(16)$ & $2(3.8)$ & 0.05 \\
\hline Trombofilia Arteriais ou & & & & \\
\hline Venosas & $6(5.6)$ & $5(9.2)$ & $1(1.9)$ & 0.39 \\
\hline Vasculite associada & $11(10.3)$ & $7(12.9)$ & $4(7.6)$ & 1.0 \\
\hline Dissecção & $7(6.6)$ & $2(3.7)$ & $5(9.6)$ & 0.26 \\
\hline Trombose & $10(9.4)$ & $7(12.9)$ & $3(5.7)$ & 0.32 \\
\hline
\end{tabular}

Procedemos à construção de uma matriz de variáveis e dividimos a população coletada entre aqueles que tinham ou não CDE (a divisão foi realizada de acordo com a positividade do DTC e TCCS, independente do ETE). Essa divisão foi feita para analisarmos as diferenças entre os subgrupos e o comportamento das variáveis entre eles.

Em relação aos fatores de risco, podemos observar que HAS foi mais frequente nos pacientes que não tinham $\operatorname{CDE}(67 \%$ vs $46,2 \%$; $=0,03)$. Não foram observadas diferenças proporcionais entre diabetes, obesidade, tabagismo, etilismo, migranea e sorologia positiva doença de Chagas (relativamente baixa) entre os dois subgrupos. 
O uso de anticoncepcional ou Terapia Hormonal foi mais frequente em mulheres com CDE $(16 \%$ vs $3,8 \%, p=0,05)$.

Trombofilias Arteriais ou Venosas foram diagnosticados em 6 pacientes da amostra, 5 deles com CDE. Os diagnósticos recebidos foram: 3 pacientes com Deficiência combinada de proteína C e S, 3 pacientes com SAAF e LES.

O diagnóstico de vasculite foi confirmado em 11 pacientes, sendo 7 com CDE e 4 sem CDE. Esses pacientes fizeram arteriografia e angioressonância arterial e venosa, além dos exames laboratoriais confirmatórios. Dos 7 pacientes com CDE: 1 paciente do sexo feminino tinha tromboangeíte obliterante e era tabagista (teve 2 eventos cerebrovasculares isquêmicos), 4 pacientes com diagnóstico de SAAF após evento cerebrovascular (mulheres, com história de abortos e uso de anticoncepcional oral ou terapia de reposição hormonal), 2 pacientes tinham sorologia positiva para HIV (1 paciente com sorologia positiva também para Sifilis) e tinham vasculite secundária. Dos 4 pacientes sem CDE: vasculite foi identificada em doenças de sobreposição (overlap) do colágeno em 1 paciente (artrite reumatóide, lupus eritematoso sistêmico, esclerodermia), 1 paciente tinha vasculite secundária ao HIV, 1 paciente tinha diagnóstico prévio de lupus eritematoso sistêmico e SAAF, 1 paciente do sexo feminino tinha arterite de Takayasu (oclusão artéria carótida interna direita e doença de arco aórtico) com evento isquêmico cerebrovascular aos 37 anos.

Dissecção foi encontrada em 7 (6.6\%) dos pacientes, 2 pacientes com CDE (dissecção da artéria carótida e artéria vertebral esquerda) e 5 sem CDE. Em relação ao mecanismo dessas dissecções, todas foram espontâneas na nossa amostra, sem eventos traumáticos concomitantes. Os vasos dissecados mais comum foram as artérias vertebrais (5 pacientes) e 2 pacientes com dissecção carotídea.

Em relação a histórico e diagnóstico de tromboses em outros sítios (venosas ou oclusões arteriais), tivemos 10 pacientes com esse histórico ( 7 pacientes com trombose de origem venosa e 3 pacientes com oclusão arterial). Desses 10 pacientes, dois pacientes apresentaram tromboses venosas durante a internação hospitalar e 1 oclusão arterial aguda após o evento cerebrovascular isquêmico. Do total, 7 (12.9\%) dos pacientes com CDE ( 6 pacientes com trombose de origem venosa e 1 paciente com oclusão arterial aguda) e dos 3 (5.2\%) dos pacientes sem CDE, tivemos 2 com histórico de trombose venosa e 1 somente com oclusão arterial aguda.

Em relação a outras peculiaridades da amostra, 1 paciente do sexo feminino com histórico de infarto agudo do miocárdio (com CDE) aos 37 anos e somente 4 pacientes com fibrilação atrial (sem CDE), sendo 1 deles com miocardiopatia chagásica aos 42 anos. 


\subsection{2 - Etiologia do AVC na população do estudo}

A classificação de TOAST utilizada encontra-se na tabela 7, onde a amostra também foi dividia em 2 subgrupos (aqueles com CDE vs sem CDE).

Pacientes com toda investigação etiológica completa, porém negativa foram considerados como AVCi criptogênico, no qual foram encontrados 29 (27.3\%) do total da amostra. Desses pacientes, a presença de CDE esteve em 23 (42\%), enquanto somente 6 $(11.5 \%)$ dos pacientes sem CDE se enquadram nessa classificação. ( $p<0.001)$.

A classificação de TOAST teve distribuição diferente entre os grupos $(p=0,003)$. Pacientes sem CDE tiveram etiologia mais frequente DCV isquêmica cardioembólica (13 vs 2) e pequenos vasos (4 vs 1). Pacientes com AVCi criptogênico foram considerados na classificação indeterminado do TOAST. Tivemos 58 (54,7\%) dos pacientes com TOAST classificado como indeterminado. Em 29 (50\%) do TOAST considerado indeterminado, os pacientes foram classificados como AVCi criptogênico, 10 pacientes tiveram duplo mecanismo de AVCi/AIT (mecanismo misto: doença de pequenos vasos, grandes vasos e cardioembólico) e 19 pacientes classificados em categoria indeterminada, a despeito de exaustiva investigação.

Tabela 7. Classificação de TOAST da população do estudo.

\begin{tabular}{|c|c|c|c|c|}
\hline \multirow[b]{2}{*}{ Variável } & \multirow[b]{2}{*}{$\begin{array}{l}\text { total } \\
\mathrm{n}=106\end{array}$} & \multicolumn{2}{|c|}{$\begin{array}{c}\text { Comunicação } \\
\text { Direita-Esquerda (CDE) }\end{array}$} & \multirow[b]{2}{*}{$\mathrm{p}$ value } \\
\hline & & $\begin{array}{l}\text { Sim } \\
n=54\end{array}$ & $\begin{array}{l}\text { Não } \\
\mathrm{n}=52\end{array}$ & \\
\hline $\begin{array}{l}\text { Criptogênico } \\
\text { (causa desconhecida) }\end{array}$ & $29(27.3)$ & $23(42)$ & $6(11.5)$ & $\mathrm{p}<0.001$ \\
\hline \multicolumn{5}{|l|}{ TOAST } \\
\hline indeterminado & $58(54.7)$ & $37(68.5)$ & $21(40.3)$ & \\
\hline outras causas & $22(20.7)$ & $11(20.3)$ & $11(21.1)$ & \\
\hline cardioembólico & $15(14.1)$ & $2(3.7)$ & $13(25)$ & 0.003 \\
\hline pequenos vasos & $5(4.7)$ & $1(1.8)$ & $4(7.6)$ & \\
\hline grandes vasos & $6(5.6)$ & $3(5.5)$ & $3(5.7)$ & \\
\hline
\end{tabular}




\subsection{3 - Frequência dos exames realizados nos pacientes (TCCS, ETE, DTC)}

Os gráficos abaixos demonstram a frequência dos exames e a porcentagem dos pacientes submetidos a TCCS, ETE e DTC (gráfico 1). O gráfico 2 demonstra o número de pacientes que realizou os dois exames (ETE e TCCS) e ( DTC e TCCS) para pesquisa de FOP e CDE. Os resultados e o cálculo estatístico dos métodos diagnósticos são baseados nos números apresentados nesses gráficos. Assim, dos 106 pacientes do estudo, 104 (98\%) realizaram o ETE, 87 (82\%) realizaram o DTC e 100 (94\%) realizaram o TCCS.

Gráfico 1. Porcentagem dos pacientes da amostra que realizaram os exames de investigação para FOP e CDE.

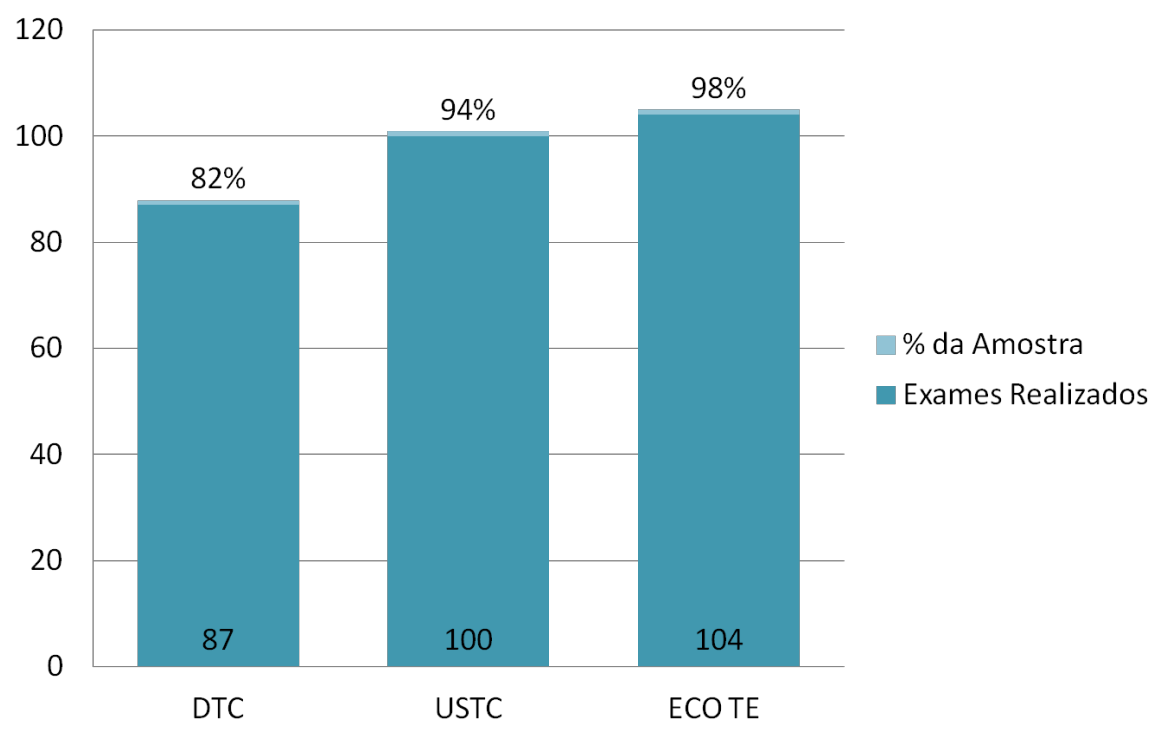

Legendas: USTC: TCCS: Ultrassonografia Transcraniana Colorida, DTC: Doppler Transcraniano, ECO TE $=$ ETE $:$ Ecocardiografia Transesofágica . 
Gráfico 2. Porcentagem de pacientes da amostra que fizeram ETE e ao menos um dos exames ultrassonográficos transcraniano para pesquisa de FOP ou CDE.

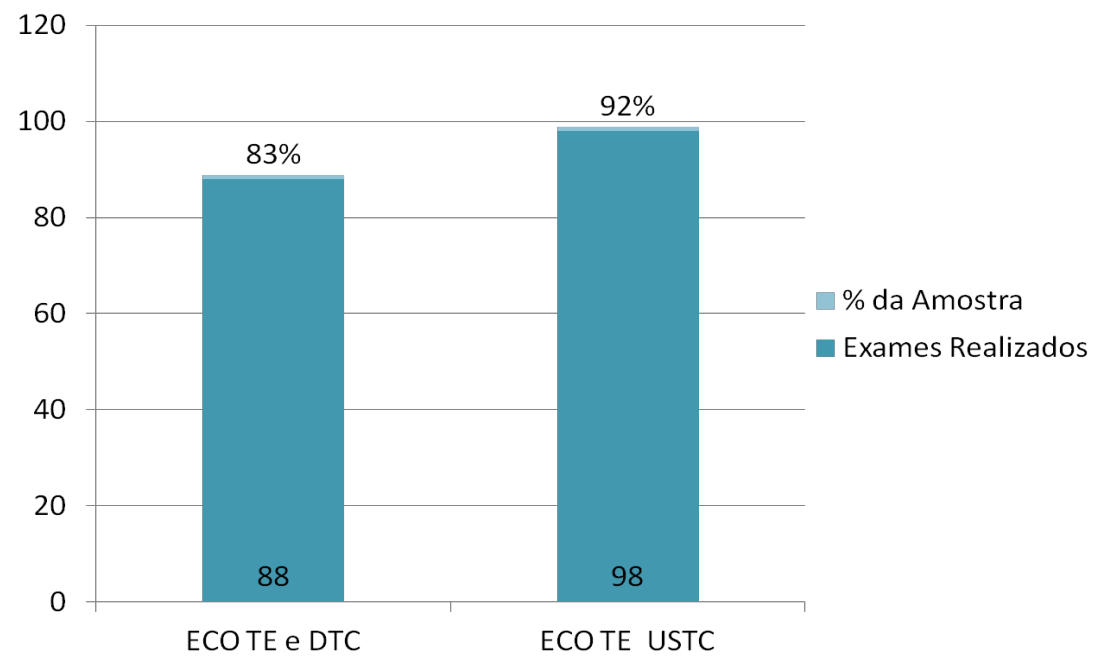

Legendas: $\mathrm{ECOTE}=\mathrm{ETE}$ e USTC $=$ TCCS.

\subsection{4 - TCCS vs ETE para diagnóstico de FOP}

Os dados compilados nas tabelas 8 e 9 demonstram a correspondência da positividade do TCCS e do ETE, considerando o ETE como padrão ouro para o diagnóstico de FOP. Os exames considerados positivos, no TCCS e no ETE são considerados verdadeiros positivos. Três pacientes com TCCS positivo, tiveram ETE negativo, em exames de Angiotomografia de Tórax para confirmação de comunicação extra-cardíaca ou fístula pulmonar, não houve a confirmação de tal achado. Nenhum paciente do estudo foi diagnosticado com Fístula Pulmonar ou Comunicação extra-cardíaca.

Tabela 8. Número de pacientes com exame TCCS positivo/negativo e correspondência com o ETE.

\begin{tabular}{|c|c|c|c|c|}
\hline & ETE & Positivo & Negativo & total \\
\hline \multirow[t]{3}{*}{ TCCS } & Positivo & 23 & 20 & 43 \\
\hline & Negativo & 3 & 52 & 55 \\
\hline & total & 26 & 72 & 98 \\
\hline
\end{tabular}

1: exame positivo 0: exame negativo. Total: total de exames positivos ou negativos. Correspondência com FOP. 
Tabela 9. Acurácia entre USTC e ETE.

\begin{tabular}{|c|c|c|}
\hline Estimativa & Estimativa (\%) & IC 95\% (LI-LS) \\
\hline Sensibilidade & $88.4 \%$ & $0.68-0.97$ \\
\hline Especificidade & $72.2 \%$ & $0.60-0.81$ \\
\hline Valor Preditivo Positivo & $53.4 \%$ & $0.39-0.67$ \\
\hline Valor Preditivo Negativo & $94.5 \%$ & $0.90-0.98$ \\
\hline Likelihood Ratio $+(\mathrm{LR}+)$ & 3.18 & $2.14-4.73$ \\
\hline Likelihood Ratio - (LR-) & 0.16 & $0.054-0.46$ \\
\hline
\end{tabular}

\subsection{5 - DTC vs ETE para diagnóstico de FOP}

Os dados compilados nas tabelas 10 e 11 demonstram a correspondência da positividade do DTC e do ETE, considerando o ETE como padrão ouro para o diagnóstico de FOP. Os exames considerados positivos, no DTC e no ETE são considerados verdadeiros positivos. Três pacientes com DTC positivo, tiveram ETE negativo, em exames de Angiotomografia de Tórax para confirmação de comunicação extra-cardíaca ou fístula pulmonar, não houve a confirmação de tal achado. Nenhum paciente do estudo foi diagnosticado com Fístula Pulmonar ou Comunicação extra-cardíaca. 
Tabela 10. Número de pacientes com exame DTC positivo/negativo e correspondência com o ETE

\begin{tabular}{|c|c|c|c|c|}
\hline & ETE & Positivo & Negativo & total \\
\hline \multirow{3}{*}{ DTC } & Positivo & 19 & 13 & 32 \\
\hline & Negativo & 3 & 53 & 56 \\
\hline & total & 22 & 66 & 88 \\
\hline
\end{tabular}

1: exame positivo 0: exame negativo. Total: total de exames positivos ou negativos. Correspondência com FOP.

Tabela 11. Acurácia entre Doppler Transcraniano (DTC) e Ecocardiografia Transesofágica (ETE)

$$
\text { Estimativa (\%) IC 95\% (LI - LS) }
$$

\begin{tabular}{lcc}
\hline Sensibilidade & 86.3 & $0.68-0.88$ \\
Especificidade & 80.3 & $0.43-0.74$ \\
Valor Preditivo Positivo & 59.3 & $0.43-0.74$ \\
Valor Preditivo Negativo & 94.6 & $0.90-0.98$ \\
Likelihood Ratio + (LR+) & 4.38 & $2.62-7.33$ \\
Likelihood Ratio - (LR-) & 0.16 & $0.058-0.48$ \\
\hline
\end{tabular}

\subsection{6 - TCCS vs DTC para pesquisa de CDE}

Os dados compilados nas tabelas 12 e 13 demonstram a correspondência da positividade do TCCS e do DTC, considerando o DTC como padrão ouro para o diagnóstico de CDE. Os exames considerados positivos, no DTC e no TCCS são considerados verdadeiros positivos. Dois pacientes com TCCS positivo para CDE e negativo no DTC confirmaram existência de FOP no ETE, esses pacientes tiveram MES ou HITS $>10$ e não detectados pelo DTC. 
Tabela 12. Número de pacientes com exame TCCS positivo/negativo e correspondência com o ETE.

\begin{tabular}{|c|c|c|c|c|}
\hline \multicolumn{5}{|c|}{ DTC } \\
\hline & & Positivo & Negativo & Total \\
\hline \multirow[t]{3}{*}{ TCCS } & Positivo & 33 & 2 & 35 \\
\hline & Negativo & 0 & 52 & 52 \\
\hline & Total & 33 & 54 & 87 \\
\hline
\end{tabular}

Legenda: Total: total de exames positivos ou negativos. Correspondência com FOP.

1: exame positivo 0 : exame negativo.

No teste de concordância entre os métodos do DTC e TCCS inter-observadores foi observado um coeficiente de Kappa de Cohen de 0.92 (IC 95\% 0.78-1.0).

Tabela 13. Acurácia entre DTC e TCCS

Estimativa (\%) IC 95\% (LI - LS)

\begin{tabular}{lcc}
\hline Sensibilidade & $100 \%$ & $0.89-1.0$ \\
Especificidade & $96.2 \%$ & $0.87-0.98$ \\
Valor Preditivo Positivo & $94.2 \%$ & $0.83-0.97$ \\
Valor Preditivo Negativo & $100 \%$ & $0.88-1.0$ \\
& & $6.93-105.2$ \\
\hline
\end{tabular}




\section{Discussão}

Pacientes jovens ( $<55$ anos) possuem maior frequência de FOP quando comparados ao grupo controle em diversos estudos. Em nossa casuística, encontramos um percentual de CDE em 54 (50.9\%) dos pacientes. Tabela 14.

Tabela 14. Estudos na literatura que comprovam que a frequência de FOP na população com DCV isquêmica, no caso AVCi ou AVEi é maior no grupo controle. ${ }^{3}$

\begin{tabular}{lccccccr}
\hline Autores & Ano & Grupo & Grupo & Método & Característica da & FOP & FOP \\
& & Estudo & controle & & amostra & Estudo & controle \\
\hline Lechat & 1988 & 60 & 100 & ETTc & AVEi $<55$ a & 40 & 10 \\
Lechat & 1988 & 26 & 100 & ETTc & AVEic $<55$ a & 54 & 10 \\
Webster & 1988 & 40 & 40 & ETTc & AVEic $<40$ a & 50 & 15 \\
Cabanes & 1993 & 100 & 50 & ETEc & AVEi $<55$ a & 43 & 18 \\
Cabanes & 1993 & 64 & 50 & ETEc & AVEic $<55$ a & 56,3 & 18 \\
Fisher & 1995 & 92 & 908 & ETEc & AVEi & 10 & 8,7 \\
Di Tullio & 2007 & 164 & 936 & ETTc & AVEic $>39$ a & 18,2 & 21,2 \\
\hline
\end{tabular}

NOTA: $\mathrm{n}=$ amostra; AVEi = acidente vascular encefálico isquêmico; $\mathrm{AVEic}=$ acidente vascular encefálico isquêmico criptogênico; $\mathrm{ETEc}=$ ecocardiograma transesofágico contrastado; $\mathrm{ETTc}=$ ecocardiograma transtorácico contrastado; FOP $=$ forame oval patente

Em revisão de literatura, nota-se que o DTC já demonstrou uma boa acurácia e como uma ferramenta segura de triagem para FOP. Todos esses estudos consideraram o ETE como exame padrão-ouro. No presente estudo, encontramos sensibilidades e especificidades, $86,3 \%$ e $80.3 \%$, indicando que o DTC é uma boa ferramenta de triagem nos pacientes com AVCi ou AIT. (Tabela 15).

Tabela 15. Estudos que demonstram a acurácia e utilizaram diferentes soluções de contraste para o exame de DTC. 


\begin{tabular}{|c|c|c|c|c|c|c|c|c|c|}
\hline \multirow{3}{*}{ Autores, Ano } & \multirow{3}{*}{$\mathrm{n}$} & \multicolumn{2}{|c|}{ Metodologia } & \multirow{3}{*}{$\begin{array}{l}\text { ETEc+ / } \\
\text { DTCc+ }\end{array}$} & \multirow{3}{*}{$\begin{array}{l}\text { ETEc- / } \\
\text { DTCc- }\end{array}$} & \multirow{3}{*}{$\begin{array}{l}\text { ETEC+ } \\
\text { / DTCc- }\end{array}$} & \multirow{3}{*}{$\begin{array}{l}\text { ETEc- / } \\
\text { DTCc+ }\end{array}$} & \multirow{3}{*}{$\begin{array}{c}\text { Sensib } \\
.\end{array}$} & \multirow{3}{*}{$\begin{array}{c}\text { Especif } \\
.\end{array}$} \\
\hline & & DTCC & & & & & & & \\
\hline & & Solução & MV & & & & & & \\
\hline Klötzsch, 1994 & 111 & Echovist $^{(2)}$ & $\mathrm{NI}$ & 42 & 61 & 4 & 4 & 91 & 94 \\
\hline Job, 1994 & 137 & Gelatina & Pós & 58 & 66 & 7 & 6 & 89 & 92 \\
\hline Horner, 1997 & 45 & Echovist $^{(\boxplus)}$ & Pós & 34 & 7 & 3 & 1 & 92 & 87 \\
\hline Nygren, 1998 & 21 & Gelatina & $\mathrm{NI}$ & 10 & 9 & 0 & 2 & 100 & 82 \\
\hline Droste, 2000 & 58 & Echovist $^{(\boxplus)}$ & Pós & 21 & 21 & 0 & 16 & 100 & 57 \\
\hline Negrão, 2005 & 75 & $\mathrm{NI}$ & $\mathrm{NI}$ & 29 & 40 & 5 & 1 & 85 & 97 \\
\hline Souteyrand, 2006 & 110 & SSA & Pós & 42 & 64 & 0 & 4 & 100 & 94 \\
\hline Sastry, 2009 & 39 & SSAs & $\mathrm{NI}$ & 16 & 10 & 0 & 13 & 100 & 43 \\
\hline Kobayashi, 2009 & 321 & SSA & Pré & 46 & 213 & 59 & 3 & 44 & 99 \\
\hline
\end{tabular}

NOTA: $\mathrm{n}$ = amostra; DTCc = Doppler transcraniano contrastado; Especif: especificidade; ETEc = ecocardiograma transesofágico contrastado; ETEc+: FOP pelo ETEc; ETEc-: ausência de FOP pelo ETEc; DTCc+: FOP pelo DTCc; DTCc-: ausência de FOP pelo DTCc; MV: manobra de Valsalva; NI: nāo informado; Pós = manobra sensibilizadora após a injeçâo de soluçāo contrastante; Pré = manobra sensibilizadora antes da injeçāo de solução contrastante; seg = segundos; Sensib: sensibilidade; SSA = soluçāo salina agitada; SSAs: soluçāo salina agitada com sangue.

É possível observar que na maioria dos estudos, o DTC demonstrou uma boa sensibilidade e especificidade principalmente na realização da MV. O último estudo citado na tabela demonstrou baixa sensibilidade, porém a MV foi realizada antes da injeção da solução salina agitada. Isso demonstra a importância de realizar a MV após o contraste. Tal estudo comparando com outros foi de fundamental importância para estabelecer a técnica de MV em nosso laboratório. ${ }^{3}$

Em relação ao TCCS comparando com o ETE, poucos estudos foram publicados de maneira sistemática na literatura (tabela 16 ).

Uma revisão na literatura desses estudos foi realizada com os valores de acurácia entre TCCS e ETE para FOP listados abaixo. 
Tabela 16. Acurácia do TCCS com o ETE em diferentes estudos.

\begin{tabular}{|c|c|c|c|c|c|c|c|c|c|c|c|}
\hline AUTORES & ANO & REVISTA & $\mathrm{N}$ & $\begin{array}{c}\text { Metodologia } \\
\text { USTC (sol } \\
\text { usada) }\end{array}$ & $\begin{array}{c}\text { ECO TE } \\
+/ \\
\text { USTC + }\end{array}$ & $\begin{array}{c}\text { ECO TE } \\
+/ \text { USTC } \\
-\end{array}$ & $\begin{array}{l}\text { ECO TE } \\
\text {-USTC + }\end{array}$ & $\begin{array}{c}\text { SEN } \\
\text { S }\end{array}$ & & \multicolumn{2}{|c|}{ ESP VPP VPN } \\
\hline & & ULTRASSOU & & & 220 & & & & & & \\
\hline Komar, M & 2014 & ND & 420 & SALINAAR & (52.3) & $21(5.0)$ & $20(4.8)$ & 95 & 92 & 98 & 89 \\
\hline Blersch, W & 2002 & Radiology & 40 & galactose & $21(52)$ & $2(5.0)$ & $2(5)$ & 91 & 88 & 91 & 88 \\
\hline $\begin{array}{l}\text { Mangiafico, } \\
\text { S }\end{array}$ & 2009 & $\begin{array}{c}\text { J Cardiovasc } \\
\text { Med }\end{array}$ & 286 & SALINAAR & $156(54)$ & $10(3)$ & $4(1)$ & 94 & 97 & 98 & 92 \\
\hline
\end{tabular}

Legendas: USTC; Ultrassonografia Transcraniana Colorida ou TCCS / ECO TE $=$ ETE Ecocargiografia Transesofáfica / Sol: solução (utilizada para realização da Manobra de Valsalva) Sensib = sensibilidade / Especif $=$ Especificidade $/$ VPP $=$ Valor Preditivo Positivo $/$ VPN $=$ Valor Preditivo Negativo. Fonte: Pubmed Libardi et al, 2015.

Em nossa casuística, encontramos uma Sensibilidade e Especificidade de $88.4 \%$ e $72.2 \%$, respectivamente, o que demonstra boa acurácia do exame de TCCS com ETE.

Nenhum desses estudos demonstrou realização de TCCS e DTC e estudou a concordância entre os exames, considerando em nosso estudo, um Kappa de Cohen de 0.92 com excelente concordância, visto que os examinadores foram cegos para ambos os exames. 


\section{Conclusões}

Os exames de TCCS e ETE possuem uma excelente acurácia para o diagnóstico de FOP e o TCCS pode ser utilizado como ferramenta de screening para essa condição.

$>$ O TCCS é uma boa ferramenta de screening para CDE com excelente concordância e boa acurácia com o DTC.

> Pacientes jovens com DCV isquêmica com CDE possuem menos fatores de risco, como HAS e DM, porém mulheres que com CDE e AVCi ou AIT possuem maior risco quando utilizam terapias hormonais (principalmente uso de anticoncepcional oral combinado). Pacientes considerados jovens ( $<55$ anos) com AVCi ou AIT possuem múltiplos fatores de risco para DCV isquêmica, o que configura um problema também epidemiológico em uma população economicamente ativa.

\subsection{Conclusões Adicionais}

$>$ Serão necessários estudos com maior amostragem e em diversos centros que utlizam a ferramenta do TCCS, ETE e DTC para screening de FOP e CDE, com necessidade de verificar a concordância inter examinadores.

$>$ A quantificação do CDE pelo DTC e TCCS deve ser considerada para comparação do tamanho da CDE, principalmente inerente ao FOP e considerar planejamento terapêutico com prevenção secundária adequados para evitar novas recorrências. Trials que consideram o fechamento do FOP na população com AVCi criptogênico deverão ser publicados e considerados em futuros guidelines para manejo terapêutico e cirúrgico dessa condição nessa população. 


\section{Referências}

1. Drighil A, EL Mosalami, H, Elbadaoui N, Chraibi S, Bennis A. Patent foramen ovale: a new disease? International Journal of Cardiology, 2007; 122: 1-9.

2. Wongpraparut N, Zaman M, Langan N, Jacobs LE, Koetler MN, Owen AN. Paradoxical embolism. Echocardiography, 2002; 19: 77-78.

3. Lange MC, Zétola VF,Souza AM, Piovesan EJ, Muzzio JA, Germiniani FGB, Werneck LC. Transcranial Doppler for Patent Forame Ovale Screening - is there a good correlation with transesophageal echocardiography. Arq Neuropsiquiatr 2008, $66(4) ; 785-789$.

4. Hagen PT, Scholz DG, Edwards WD. Incidence and size of patent foramen ovale during the first 10 decades of life: an autopsy study of 965 normal hearts. Mayo Clin Proc 1984; 59:17-20.

5. Lynch JJ, Schuchard GH, Gross CM, Wann LS. Prevalence of right-to-left atrial shunting in a healthy population: detection by Valsalva maneuver contrast echocardiography.Am J Cardiol 1984; 53:1478-1480.

6. Konstadt SN, Louie EK, Black S, Rao TLK, Scanlon P. Intraoperative detection of patent foramen ovale by transesophageal echocardiography.Anesthesiology 1991; $74: 212-216$.

7. Fisher DC, Fisher EA, Budd JH, Rosen SE, Goldman ME. The incidence of patent foramen ovale in 1000 consecutive patients: a contrast transesophageal echocardiography study. Chest 1995; 107:1504-1509.

8. Cabanes L, Mas Jl, Cohen A, et al. Atrial septum aneurysm and patent foramen ovale as risk factors for cryptogenic stroke in patients less than 55 years of age. Stroke $1993 ; 24: 1865-1873$. 
9. Hausmann D, Mügge A, Becht I, Daniel WG. Diagnosis of patent foramen ovale by transesophageal echocardiography and association with cerebral and peripheral embolic events. Am J Cardiol 1992; 70:668-672.

10. Lechat P, Mas JL, Lascault G, et al. Prevalence of patent foramen ovale in patients with stroke. N Engl J Med 1988; 318: 1148-1152.

11. Webster MW, Chancellor AM, Smith HJ, et al. Patent foramen ovale in young stroke patients. Lancet 1988; 2:11-12.

12. Di Tullio M, Sacco RL, Gopal A, Mohr JP, Homma S. Patent foramen ovale as a risk factor for cryptogenic stroke. Ann Intern Med 1992; 117:461-465.

13. De Castro S, Cartoni D, Fiorelli M, et al. Morphological and functiona characteristics of patent foramen ovale and their embolic implications. Stroke 2000; 31:2407-2413.

14. Homma S, Di Tullio MR, Sacco RL, Mihalatos D, Li Mandri G, Mohr JP. Characteristics of patent foramen ovale associated with cryptogenic stroke. Stroke 1994; 25: 582-586.

15. Serena J, Segura T, Perez-Ayuso MJ, Bassaganyas J, Molins A, Davalos. The need to quantify right-to-left shunt in acute ischemic stroke. Stroke 1998; 29:1322-1328.

16. Soliman OI, Geleijnse ML, Meijboom FJ, Nemes A, Kamp, O Nihoyannopoulos P, Masani N.; Feinstein SB, Ten Cate FJ. The use of contrast echocardiography for the detection of cardiac shunts. European Journal of Echocardiography, 2007; 8: S2-S12.

17. Cabanes L, Coste J, Derumeaux G, Jeanrenaud X, Lamy C, Zuber M, Mas JL. Interobserver and intraobserver variability in detection of patent foramen ovale and atrial septal aneurysm with transesophageal echocardiography. Journal of American Society of Echocardiography, 2002;15: 441-446. 
18. Blersch WK, Draganski BM, Holmer S, Kock HS, Schlachetzki F, Bogdahm U, Holscher T. Transcranial Duplex Sonography in the detection of patent foramen ovale. Radiology 2002 225: 693 - 699.

19. Jauss M, Zanette E. Detection of Right-to-Left Shunt with ultrasound contrast agent and Transcranial Doppler Sonography. Cerebrovascular Diseases. 2000;10(6):490496.

20. Sastry S, Daly K, Chengodu T, McCollum C. Is transcranial Doppler for the detection of venous-to-arterial circulation shunts reproducible? Cerebrovasc Dis 2007;23:424-429.

21. Sacco RL, Toni D, Brainin M, MOHR JP. Classification of ischemic stroke. (Ed). Stroke: pathophysiology, diagnosis and management. 4a ed. Philadelphia, PA: Churchill Livingstone, 2004. p. 61-74.

22. Del Sette M, Dinia L, Rizzi D, Sugo A, Albano B, Gandolfo C. Diagnosis of right-toleft shunt with transcranial Doppler and vertebrobasilar recording. Stroke 2007;38:2254-2256.

23. Perren F, Savva E, Landis T. Trasforaminal Doppler: An alternative to transtemporal approach for right-to-left cardiac shunt assessment. J Neurol Sci 2008;273:49-50.

24. Tsivgoulis G, Neumyer MM, Alexandrov AV. Diagnostic criteria for cerebrovascular ultrasound. In: Alexandrov A (ed). Cerebrovascular Ultrasound in Stroke Prevention and Treatment, 2nd Ed. New York: Blackwell Publishing, 2011, p. 85-143.

25. Lao AY, Sharma VK, Tsivgoulis G, et al. Detection of Right - to- Left Shunts: Comparison between the International Consensus and Spencer Logarithmic Scale Criteria. Journal of Neuroimaging. 2008:402-406. 
26. Muzzio, JA; Zetola, VF; Lange, MC. Embolia paradoxal. In: Editores: Viviane H. Flumignan Zetola e Marcos Christiano Lange. Manual de Doppler Transcraniano da Academia Brasileira de Neurologia - DC de Doppler Transcraniano, 2006, p. 89-93.

27. Spencer MP, Moehring MA, Jesurum J, et al. Power M-Mode Transcranial Doppler for Diagnosis of Patent Foramen Ovale and Assessing Transcatheter Closure. Journal of Neuroimaging. 2004;(4):342-349.

28. Topcuoglu MA, Palacios IF, Buonanno FS. Contrast M-mode power Doppler ultrasound in the detection of right-to-left shunts: utility of submandibular internal carotid artery recording. J Neuroimaging 2003;13:315-23

29. Ringelstein EB, Droste DW, Babikian VL, et al. Consensus on Microembolus Detection by TCD International Consensus Group on Microembolus Detection. Stroke. 1998:725-729.

30. Marinoni M, Ginanneschi A, Forleo P, Amaducci L. Technical limits in transcranial Doppler recordings: inadequate acoustic window. Ultrasound Med Biol 1997;23:1275-1277.

31. Wijnhoud AD, Franckena M, van der Lugt A, Koudstaal PJ, Dippel ED. Inadequate acoustical temporal bone window in patients with a transient ischemic attack or minor stroke: Role of skull thickness and bone density. Ultrasound Med Biol 2008;34:923929.

32. Censori B, Partziguian T, Poloni M. Common carotid artery duplex for the bubble test to detect right-to-left shunt. J Ultrasound Med 2010; 36:556-570.

33. Venketasubramanian N, Sacco RL, DI Tullio M, Sherman D, Homma S, Mohr JP. Vascular distribution of paradoxical emboli by transcranial Doppler. Neurology, 1993; 43: 1533-1535. 
34. Draganski B, Blersch W, Holmer S, Koch H, May A, Bogdahn U, Ho“lscher T, Schlachetzki F. Detection of cardiac right-to-left shunts by contrast-enhanced harmonic carotid duplex sonography. J Ultrasound Med 2005;24:1071-1076.

35. Adams HPJr, Bendixen BH, Kapelle LJ, Biller J, Love BB, Gordon DL, Marsh EE. Classification of subtype of acute ischemic stroke. Definitions for use in a multicenter clinical trial TOAST - Trial of Org 10172 in Acute Stroke Treatment. Stroke1993 Jan;24(1):35-41. 


\section{Anexos}

ANEXO A -Termo de Consentimento Livre e Esclarecido

TERMO DE CONSENTIMENTO LIVRE E ESCLARECIDO - TCLE

hospital das Clínicas da Faculdade de Medicina de Ribeirão Preto UNIVERSIDAdE DE SÃo PAULO (FMRP-USP)

\section{Pesquisa: Acurácia da Ultrassonogorafia Transcraniana Colorida no Diagnótico de Forame Oval Patente \\ Pesquisadores responsáveis: \\ Milena Carvalho Libardi - contato: (14) 99718-2178 \\ Octávio Marques Pontes-Neto - contato: (16) 98101-3465}

Gostaríamos de convidá-lo(a) a participar de uma pesquisa sobre "Acurácia da Ultrassonografia Transcraniana Colorida no Diagnóstico de Forame Oval Patente". O acidente vascular cerebral ou "derrame" é uma doença séria que é causada pelo entupimento (acidente vascular cerebral isquêmico) ou ruptura (acidente vascular cerebral hemorrágico) do vaso sanguíneo cerebral.

O Forame Oval Patente (FOP) é uma comunicação proveniente do período de formação fetal cardíaca que permite a passagem direta do sangue do lado direito para o lado esquerdo do coração. Esse orifício cria diferenças de pressão entre um sangue venoso pouco oxigenado e um sangue arterial oxigenado originando pequenos coágulos denominados de microêmbolos que podem passar para a circulação cerebral através dos vasos cervicais e causar o "derrame". Logo, a investigação do FOP é de suma importância e pode auxiliar na descoberta da causa do "derrame" e contribuir para que os médicos tracem planos e estratégias para impedir recorrência ou novos eventos cerebrovasculares isquêmicos. Através desse estudo, queremos realizar pesquisa desses pequenos êmbolos nos pacientes com "derrame" através da realização de um exame ultrassonográfico da cabeça e do pescoço, o Duplex e o Doppler Transcraniano. Trata-se de uma exame tipo "ultra-som", de simples execução. Para a pesquisa do FOP haverá necessidade de punção em veia periférica do braço e a solução contendo somente soro fisiológico será injetada. Pode raramente ocorrer dor, infecção e/ou/hematomas no local da punção, sendo a solução isotônica isenta de riscos.Tal procedimento é considerado rotina no serviço. Solicitamos, também, sua autorização para analisarmos outros exames que são feitos, de rotina, em nosso serviço, como a Ecocardiografia Transesofágica. Apesar de não mudar o seu tratamento em nada, essa pesquisa verificará se o exame de Ultrassonografia (Duplex)e o Doppler são capazes de ajudar na investigação de forame oval patente, possibilitando um maior número de diagnóstico. Assim, permitirá que orientações específicas sejam feitas para que se tente evitar novos eventos cerebrais isquêmicos. As informações obtidas são sigilosas e confidenciais e só serão utilizadas para fins de pesquisa. Qualquer intercorrência relacionada ao procedimento desta pesquisa será de responsabilidade do investigador responsável e você terá toda assistência médica 
necessária nesta instituição até a solução deste problema. Essa pesquisa não acarretará despesas a você e nem a sua família e você deverá receber uma cópia desse termo assinada e rubricada pelo pesquisador responsável em todas as páginas, deverá também rubricar todas as páginas do mesmo. Qualquer informação adicional poderá ser fornecida em qualquer fase da pesquisa pelo pesquisador abaixo relacionado. Você e sua família estão livres para decidir se querem ou não participar desta pesquisa e sua decisão não mudará a forma como vocês serão atendidos neste Hospital. Vocês também estão livres para sair da pesquisa a qualquer momento que quiserem, sem nenhum problema ou prejuízo no tratamento e qualquer esclarecimento ou dúvidas inerentes a pesquisa podem ser esclarecidas pela pesquisadora responsável Dra Milena Carvalho Libardi (telefone: 14 997182178).Esse projeto também consta no Comitê de Ética em Pesquisa do Hospital das Clínicas e da Faculdade de Medicina de Ribeirão Preto, cujo telefone é (16) 3602-22228.

Declaro por meio desta, que fui devidamente esclarecido dos objetivos, métodos e justificativas da pesquisa intitulada "Acurácia da Ultrassonografia Transcraniana Colorida no Diagnóstico do Forame Oval Patente" Diante do exposto, declaro que concordo em participar desta pesquisa de forma livre e esclarecida de seu conteúdo, sabendo que posso dela sair no momento em que desejar, sem que isto implique em qualquer prejuízo do tratamento.

Nome do participante da pesquisa

Assinatura do Participante da Pesquisa

Nome do responsável legal

Assinatura do Responsável Legal

Grau de parentesco/ relação social

Nome do pesquisador responsável

Assinatura do Pesquisador Responsável
RG

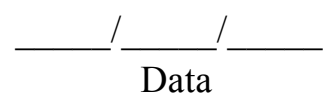

RG

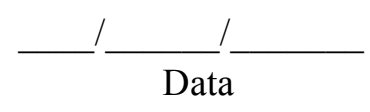




\section{ANEXO B}

\section{Resumo dos trabalhos publicados em anais de Congresso}

Abaixo estão os abstracts/resumos dos trabalhos enviados ao Congresso Brasileiro de Doenças Cerebrovasculares no mês de outubro de 2015 na cidade de Belo Horizonte, Minas Gerais. (Dois trabalhos como apresentação oral/ tema livre) e o trabalho que será enviado Congresso Americano (Stroke International Conference) em Los Angeles, Califórnia em fevereiro de 2016 como pôster.

\section{Congresso Brasileiro de Doenças Cerebrovasculares 2015}

Local: Minas Centro, Belo Horizonte

Modalidade: Apresentação Oral

Evaluation of Risk Factors in Brazilian Young with Stroke and the relation with the presence of right-to-left shunt

Author Block Milena C. Libardi, Millene R. Camilo, Soraia R. Fabio, Rui Kleber V. Martins Filho, Letícia J. Rocha, Renata S. Santos, Clara M. Barreira, Pedro T. Cougo-Pinto, Frederico F. Alessio-Alves, Francisco A. Dias, Daniel G. Abud, Octávio M. Pontes-Neto.

Introduction: Right-to-left shunts (RLS) are found in young patients with ischemic stroke, being Persistent Foramen Ovale (PFO) the most common shunt. The RLS is the mechanism of paradoxical embolism and is enhanced by prothrombotic conditions but when consider RLS incidental or stroke related remains a big challenge. Objectives: Evaluate the role of RLS in young Brazilian patients with ischemic stroke through the risk factors and prothrombotic conditions. Methods: 106 patients with ischemic stroke under 55 years-old were admitted in our tertiary university hospital from 2012 to 2014 divided all in two subgroups (with RLS and no RLS) according to results of Transcranial Doppler (TCD). Risk factors for stroke are checked (Hypertension, Diabetes, Dyslipidemia, cigarette use, alcoholism, obesity), migraine, contraceptives oral use, arterial dissections, previous thrombosis, vasculitis, thrombophilia and Chagas disease considered. Results: $54(51 \%)$ patients had positive RLS in TCD, mean age 43.9 (SD:8.2) and 52 (49\%) patients had negative RLS, mean age 45.3(SD:8.7). In positive RLS group, 36/54 (66.7\%) were female. Hypertension and diabetes were negative in RLS group: 67\% $(\mathrm{p}<0.05)$ and $25 \%(\mathrm{p}=0.13)$, respectively versus $46.2 \%$ hypertension and $12.9 \%$ diabetes in negative RLS. Contraceptive oral use in $16 \%$ women in positive RLS versus $3.8 \%$ negative RLS ( $\mathrm{p}=0.05$ ). Thrombophilia variants presented in 5 patients with RLS and one patient without RLS ( $p=0.39$ ). RLS had 
more migraine $(\mathrm{n}=13 \times \mathrm{n}=7)$. Previous thrombosis was present in 7 positive RLS and 3 negative RLS $(p=0.32)$. Other factors did not present differences between groups. Cryptogenic strokes were present in $42 \%$ of positive RLS and in $11.5 \%$ of negative RLS $(\mathrm{p}<0.001)$. Conclusion: Cryptogenic strokes and prothrombotic conditions are often in RLS group, revealing that these conditions could be related about the mechanism of ischemic disease in our population and the importance of consider RLS screening in Brazilian young with prothrombotic conditions.

\section{Congresso Brasileiro de Doenças Cerebrovasculares 2015}

Local: Minas Centro, Belo Horizonte

Modalidade: Apresentação Oral

Transcranial-Color-Coded Sonography for the detection of right-to-left shunt in young stroke patients

Author Block Milena C. Libardi, Millene R. Camilo, Soraia R. Fabio, Rui Kleber V. Martins Filho, Letícia J. Rocha, Renata S. Santos, Clara M. Barreira, Pedro T. Cougo-Pinto, Frederico F. Alessio-Alves, Francisco A. Dias, Daniel G. Abud, Octávio M. Pontes-Neto.

INTRODUCTION: Right-to-left shunt (RLS) is frequently found in young patients with stroke and may suggest paradoxical embolism as the underlying stroke mechanism. Transcranial Doppler (TCD) is the standard method to screen for RLS. More recently, Transcranial-Color-Coded Sonography (TCCS), which incorporated B-mode imaging and color duplex to pulsatile Doppler, has been used for the same purpose but its accuracy has not been systematic evaluated. OBJECTIVE: To determine the accuracy of TCCS when compared to TCD for RLS diagnosis in young patients with stroke. METHODS: 87 patients with ischemic stroke under 55 years old, admitted to a tertiary academic hospital between 2013 and 2014 were submitted to a TCD exam and TCCS exam for the detection of RLS by two expert examiners that were blinded for the other results. An Interobserver Kappa agreement was calculated. The saline bubble test was performed according to international standards and right middle cerebral artery signals were through the temporal windows. Microembolic signals (MES) were counted according to International guidelines. Patients were routinely submitted to an extensive etiologic workup and classified according to the TOAST criteria. RESULTS: The mean age of the patients was $44.6 \pm 8.4$ years. According to TCD, RLS was detected in 35 (40.3\%) of patients. Twenty-nine (33.3\%) patients were diagnosed with cryptogenic stroke and RLS was detected in $23(79.3 \%)$ of them $(p<0.001)$. 
Interobserver Kappa for TCCS was 0.87 (CI 0.71-1.0). When compared to TCD, TCCS findings had $100 \%$ sensitivity, $96.7 \%$ specificity. TCCS was positive in 2 patients, in whom TCD was negative, and 3 patients did not have an appropriate transtemporal window for both technics. CONCLUSION: TCCS has excellent agreement, sensitivity and specificity when compared to TCD for RLS detection. TCCS can be accurately used for RLS screening in young patients with stroke.

\section{Congresso Americano - International Stroke Conference Los Angeles 2016}

Modalidade: Pôster

Transcranial Color Coded Sonography versus Transcranial Doppler for the detection of right-to-left shunt and Patent Foramen Ovale in young patients with stroke.

Author Block Milena C. Libardi, Millene R. Camilo, Soraia R. Fabio, Rui Kleber V. Martins Filho, Letícia J. Rocha, Renata S. Santos, Clara M. Barreira, Pedro T. Cougo-Pinto, Frederico F. Alessio-Alves, Francisco A. Dias, Daniel G. Abud, Octávio M. Pontes-Neto. Univ of São Paulo, Ribeirão Preto Medical Sch, Ribeirão Preto, Brazil

Introduction: Patent Foramen Ovale (PFO) is the most common right-to-left shunt (RLS) and is often found in young patients with stroke related to paradoxical embolism. ContrastEnhanced Transesophageal Echocardiography (TEE) is considered a gold standard to visualize PFO. Transcranial Doppler (TCD) with bubble test is often used to detect RLS with good correlation to TEE for the diagnostic of PFO. More recently, Transcranial Color Coded Sonography (TCCS) which included B-mode and color coded imaging has overcome blind TCD in many clinical applications but the accuracy of TCCS for detection of RLS and PFO has not been systematically evaluated. Hypothesis: To determine if the TCCS is an accurate tool to identify both PFO and RLS. Methods: We investigate 106 patients with ischemic stroke under 55 years-old admitted from 2012 to 2014 in a tertiary academic hospital. Patients were evaluated with TEE, TCD and TCCS, and all exams included a saline bubble test. The examiners were blinded for the other tests results. Kappa agreement was calculated inter-examiners for TCCS and TCD. Accuracy of TCCS was calculated in comparison to TEE. Results: We detected a RLS in 54 (50.9\%) patients (age mean $43.9 \pm$ 8.2) with kappa agreement 0.92 (95\%CI 0.78-1.0) when performed TCCS and TCD. TEE and TCSS were positive in 23/98 (23.4\%) and TEE did not reveal contrast in 20/98 (20.4\%). In $30(28.3 \%)$ patients only TEE revealed a PFO. TCSS had a sensitivity of $88.4 \%(95 \% \mathrm{CI}$ $0.68-0.97)$, specificity of $72.2 \%(95 \% \mathrm{CI} 0.60-0.81)$ and positive likelihood ratio of 3.18 
(2.14-4.73) of the diagnosis of PFO. Conclusion: TCCS and TCD had an excellent agreement. TCCS has a good accuracy for the detection of PFO and RLS in young patients with stroke. 